\title{
Science and the Concept of Evolution: From the Big Bang to the Origin and Evolution of Life
}

\author{
Lori Zaikowski • Richard T. Wilkens • Kurt Fisher
}

Published online: 20 November 2007

(C) Springer Science + Business Media, LLC 2007

\begin{abstract}
The common thread of evolution runs through all science disciplines, and the concept of evolution enables students to better understand the nature of the universe and our origins. "Science and the Concept of Evolution" is one of two interdisciplinary science Core courses taken by Dowling College undergraduates as part of their General Education requirements. The course examines basic principles and methods of science by following the concept of evolution from the big bang to the origin and evolution of life. Case studies of leading scientists illustrate how their ideas developed and contributed to the evolution of our understanding of the world. Evidences for physical, chemical, and biological evolution are explored, and students learn to view the evolution of matter and of ideas as a natural process of change over space and time.
\end{abstract}

Keywords Big bang · Evolution of ideas · Stellar evolution . Chemical evolution · Origin of life

\footnotetext{
L. Zaikowski $(\bowtie)$

Department of Chemistry, Dowling College,

150 Idle Hour Boulevard,

Oakdale, NY 11769, USA

e-mail: zaikows1@dowling.edu

R. T. Wilkens

Department of Biology, Dowling College,

150 Idle Hour Boulevard,

Oakdale, NY 11769, USA

e-mail: wilkensr@dowling.edu

\section{K. Fisher}

Department of Physics, Dowling College,

150 Idle Hour Boulevard,

Oakdale, NY 11769, USA

e-mail: fisherk@dowling.edu
}

\section{Introduction}

When our students hear the word "evolution", they usually think of biological evolution. Undergraduates enter our General Education science Core courses with many misconceptions about biological evolution and little understanding about the concept of evolution in general. We find that students better comprehend biological evolution when evolution is first presented in its wider context. "Science and the Concept of Evolution, NSC 2003C" integrates fundamental concepts in physics, chemistry and biology to provide students with an interdisciplinary understanding of evolution. The course chronologically explores not only the evolution of the universe, solar system and life on earth, but also the evolution of ideas regarding various realms of the scientific enterprise. The philosophy of science is introduced from the perspective of scientists in each field, and through examples of how their ideas have played out in the "evolution" of our understanding of the solar system model, the sciences of mechanics and electricity, atomic theories, and biological species change (Table 1). The course provides students with an interwoven mosaic about the evolutionary nature of our world and our understanding of that world. It follows the evolution of physical forces and of chemistry beginning with the first seconds of the universe: from the formation of the simplest elements, to more complex elements and molecules, to prebiotic compounds, and ultimately to the molecular diversity and complexity present in living organisms today. In the process, students learn how science works, how it is used to unravel the mysteries of the universe, and how physics, chemistry, and biology have evolved over time and shaped our worldview.

Dowling College is an independent, coeducational college of more than 7,000 students, approximately 3,500 of which are undergraduates. Students in all majors 
Table 1 Evolution of ideas across the sciences

\begin{tabular}{ll}
\hline Early ideas & Middle progress \\
\hline Structure of matter: atomic theory & \\
Democritus ( 530 BC) suggested & Dalton-law of definite \\
that there was an ultimate unit & proportions. Mendeleev- \\
of matter (atom - means & periodic table. J.J. Thomson- \\
"uncuttable") & discovery of electron showed \\
& existence of subatomic particles. \\
& Rutherford-nuclear atom
\end{tabular}

Physics: motion and force

Aristotle thought that constantspeed motion requires a force to maintain it. He also believed that heavier bodies fall faster than lighter ones

Chemistry: bonding and energy Phlogiston theory, Alchemy

Cosmology

Supernatural creation

Solar system and astronomy

Geocentric model based on religious beliefs, but explains observed phenomena

Earth science: geology

Earth thought to be 6,000 years old (Bible)

Hutton-Earth is old Lyellestablished geology as a science.

Galileo's experiments refuted Aristotle's theory. Newton built on Galileo's work and quantified it. Electric and magnetic forces discovered

Oxidation process discovered (Lavoisier). Basic chemical reactions known: acid-base, oxidation-reduction, bonding

Planets, stars, moons identified.

Galaxies and red shift discovered in 20th century

Careful observations (Brahe, Kepler) point to Heliocentric Model first suggested by Copernicus. Telescope confirms model
N. Bohr-explained hydrogen spectrum using quantum model of the atom. Quantum mechanics was applied to study of atom. Elementary particle theory developed but not yet complete

Newton's laws of motion and of universal gravitation hold for most human-sized objects and for speeds $<10 \%$ of lightspeed. For fast, atomic- or galaxy-sized objects, Einsteinian mechanics is necessary. Electric and magnetic forces unified by Electromagnetic Theory

Laws of Thermodynamics established. Development of novel chemical syntheses created new materials: dyes, fibers, polymers, plastics, etc.

Big Bang theory supported by discovery of cosmic background radiation, inflation theory, highenergy particle collision expts.

9 planets eventually discovered. Discovery of Neptune confirms Newton's theory of universal gravitation. Anomaly in orbit of Mercury resists solution with Newton's laws

Radioisotope dating establishes age of earth at $\sim 5$ billion years. Understanding of Earth's formation and composition.

Darwin - Origin of Species. Wallace_-independent discovery of the Theory of Evolution
Latest refinements

Relation between elementary particles and fundamental forces sought. Process of reductionism seeks to find a grand unified theory (GUT); superstring theory, $\mathrm{M}$ branes are under active research

The universal gravitational constant is hypothesized to change as the universe expands. Black holes are the ultimate condensed matter state. Strong and weak forces discovered

Ceramics, high temperature superconductors, advanced pharmaceuticals

Dark matter \& dark energy. Attempts to explain increased rate of expansion of universe.

Improvements in telescopes and spectrometers advance knowledge

Precession of Mercury's orbit is solved by Einstein's Theory of General Relativity. Theory and observations agree. Pluto is demoted to non-planet status (Resolution 6 from the XXVIth General Assembly in Prague: Definition of a Planet in the Solar System 2006)

Plate tectonics; seismology used to get more accurate picture of interior structure of earth. Early atmosphere and conditions better understood.

Discovery of the DNA double helix, RNA; the mechanism of inheritance was detailed; gene sequencing \& mapping. Prebiotic chemistry and origin of life hypotheses: Miller-Urey, RNA World, Lipid World, etc. 
complete a Core Curriculum to fulfill General Education requirements. The six-credit, two semester sequence NSC 2003C (Science and the Concept of Evolution) and NSC 2004C (The Science of Natural Systems) fulfills the Natural Sciences portion of the Core Curriculum. NSC 2003C meets for 160 min per week and 14 weeks per semester (typically $80 \mathrm{~min}$ per class meeting, 2 days per week). Over 25 sections of NSC $2003 \mathrm{C}$ are offered each year as the course is taken by virtually all Dowling undergraduates, and each section enrolls about 25 students. Most students in the course are non-science majors.

Faculty teaching the interdisciplinary NSC Core courses come from a wide variety of disciplinary backgrounds and must meet the challenge of teaching subject matter that is not in their immediate area of expertise. Pedagogical strategies vary according to each instructor's teaching style. Most instructors use a traditional lecture approach with visuals, and most ask questions in a Socratic style to foster discussion and motivate student thinking. Although the course does not have a separately scheduled laboratory, most faculty include laboratories, demonstrations, and collection and analysis of data. A library research writing assignment is required of all students. A composite sample syllabus that lists NSC 2003C course topics as well as suggested laboratories and demonstrations is available in the supplementary materials. A main objective is to impart an integrated overview of science and the concept of evolution. To achieve this integrated picture, principles of the philosophy of science are first discussed in general terms, and then are illustrated with reference to specific theories that form our contemporary view of how the world works. Likewise, when discussing specific scientific theories and how they have evolved, reference is made to the philosophical principles that were operative at the time. This bi-directional reinforcement helps to instill both the scientific principles themselves and the methods by which they came about. The narrative that follows provides a condensed introduction to the course content, describing how the course explores the nature of science, the fundamental makeup of the universe, the way in which these fundamental parts are assembled into the totality of the universe, how life on Earth evolved and fits into the cosmic fabric, and how our ideas about the universe have evolved over time. A more thorough treatment of the content knowledge can be found in the recommended texts.

\section{The Universe Alive and Kicking}

The grandest story of all encompasses the origin, makeup and evolution of our universe, which includes life. This is the story that we teach in our general education Core course "Science and the Concept of Evolution." Indeed, it is a grand story, unified by the majesty of the topic; nonetheless, there is a surfeit of compelling subplots. One subplot is a perfect complement to the overarching story - the way that scientists have come to know the universe in all its splendor and complexity. At our college educators hotly debate how best to deliver general education; we believe that this course hits many of the right notes. It encompasses topics that speak to the fundamental reality of our existence. Furthermore, it stresses the human endeavor of science. These are exactly the kinds of lessons that are important for every college student to embrace; that is, the universe is large, complex, marvelous, and often knowable. The course emphasizes what we know, how we learned it, and what is left unknown. For students to grasp the concept that scientists have fundamentally explained processes that once seemed unknowable is among the most important messages of this course. Science is alive and dynamic and the universal unknown is the frontier to which scientists set sail as explorers.

Breaking the Universe into Fundamental Parts: Matter, Force, and Time We argue that to know the universe, students should know its fundamental composition. In this unit, we break the universe into its fundamental building blocks. First, we introduce the concept of the smallest piece of matter. But to do so, we do not simply make a list of fundamental particles. Rather, we explore the subplot of discovery. How did humans come to know of the fundamental parts of matter? Starting with Democritus's átom and showing the evolution of atomic theory through a sequence of scientists (Dalton, Thomson, Rutherford, Bohr, Pauli, Fermi: Structure of Matter in Table 1) we end with our current state of knowledge: six quarks and six leptons.

Matter is not motionless, not frozen in space and time; it moves, sometimes dramatically. So next, we discuss the three laws that govern the motion of matter, courtesy of Isaac Newton. Of course, matter does not move magically; a force is responsible (Table 1 Physics). As such, we spend several lectures on the four fundamental forces, paying particular attention to gravity and electromagnetism. These two forces, along with the strong nuclear force, are essential for understanding the next unit, which covers in part, the life and death of stars. Once the concepts of matter and force have been established, we move on to the fundamentals of thermodynamics (Table 1 Chemistry). No discussion of the universe can be complete without some understanding of both laws. The first law of thermodynamics is absolutely essential to make the following point: nothing in the universe is magical; that is, whenever an object emits or utilizes energy, that energy must come from somewhere. In the next unit, this fundamental concept is used to make an important point about stars - they die. The second law of thermodynamics is no less important. It establishes a sequence of events: order goes to disorder, heat flows from 
hot to cold bodies, and energy goes from a concentrated form to a less-concentrated form with the release of heat. In other words, the second law dictates the arrow of time. Voilá, we have done it! The universe broken down into matter, force and time with a handful of laws that govern them.

Reassembling the Universe To know a thing is to know more than the fundamental building blocks. After all, if each of a human's 11 trillion cells were presented to you one at a time, you'd hardly understand what it means to be a human-so too for the universe. As such, in this unit we aim to reassemble the universe into a coherent entity made up of fundamental building blocks. The book we use for the course, The Sciences by Trefil and Hazen (2007), has a fascinating approach that is adopted for the first presentation in this unit. Imagine the first person able to form a question (perhaps one of our hominid ancestors) emerging from a slumber and looking up at the night sky. They surely asked an obvious question, "What are those points of light?" Thus, astronomy was born. In this class, the same question is asked of students, only we permit them to use the basic information learned in the previous unit. Thus follows a remarkable logical sequence: stars emit light; light is electromagnetic energy; stars are not magical, and thus, they must convert some other form of energy into light; stars are not infinite in volume, so they will run out of energy someday; all stars will die; and finally, if all stars die, then all stars were born. Two questions naturally flow from this discussion:

- How are stars born?

- How do stars die?

The answers to these questions are marvelous in their own right, but they are essential to understanding the origin of life itself, for it is in the forge of thermonuclear fusion that the elements of life were generated. By detailing the processes of star birth and death, we construct a panoply of stars: main sequence stars (from sun-sized yellow stars to blue giants), red giants, super red giants, white dwarfs, black dwarfs, neutron stars and black holes. Knowing the origin and properties of this cast of stars drives home the key point that our ideas regarding the universe's generative processes and composition have evolved as the tools available to study the universe have improved (Table 1 Cosmology and Solar System Astronomy).

As a case in point, we consider the ways that astronomers measure distances to stars. Triangulation, using trigonometric functions, to measure distance works well for stars nearby. But before powerful telescopes, there were a number of "fuzzy stars" that were far too distant to be measured using this technique (Trefil and Hazen 2007). Edwin Hubble used Cepheid variables (a special type of dying star that reveals its absolute brightness by the duration of its brightening/ dimming cycle) to determine that the so-called fuzzy stars were really clusters of stars that were astonishingly far away (Trefil and Hazen 2007); we now recognize these as galaxies. The nearest galaxy to us is Andromeda, 2.5 million light years away! Hubble helped us realize the immensity of the universe. Indeed, the arrangement of galaxies in the universe has a large-scale structure in which galaxies come in clusters and groupings. Hubble's discovery of the immensity of the universe vastly changed our sense of the universe, but he was not done surprising us. Using light spectroscopy (the same technique is performed by students in lab), Hubble discovered that all galaxies are moving away from us; the farther away the faster they move (Livio 2000). Hubble determined that only universal expansion could explain such a result! For a second time, Hubble dramatically changed the way we view the universe. Hubble's discovery emphasizes in a dramatic way that the universe is full of surprises and that scientists must be prepared to interpret them within the existing framework, or if necessary, devise new explanations.

The universe is large (infinite actually), and surprises seem to be the norm rather than the exception. In 1998, Science magazine named the discovery that the universe is not only expanding but that it is doing so at an expanding rate as its "Breakthrough of the Year" (Glanz 1998). At this point in the class, we have established how stars form and die, how they are collected into galaxies, how the galaxies are arranged in space and how they are constantly zooming away from each other at an accelerating rate! Not bad for a unit in a general science class, but the story does not end here. No; remarkably, using particle accelerators, scientists have determined with great precision what the conditions of the universe were like in the first moments after the Big Bang. For instance, at $10^{-35} \mathrm{~s}$ after the Big Bang, the strong force froze out and at $10^{-10} \mathrm{~s}$ all four fundamental forces existed as separate entities. The stunning energy and expansion of the early universe led to an important phenomenon for understanding the origin of life; that is, rapid expansion prevented the formation of heavier elements through fusion. The vast majority of matter in the universe is hydrogen, helium and lithium; everything else is rare. But hydrogen, helium and lithium simply will not suffice for life. To account for life, we need to explain the origin of heavier elements: nitrogen, oxygen, phosphorus and many others, but especially carbon. Moreover, we need to explain how they got to us here in our solar system.

Life At this point, a review session examines images from the Hubble telescope. Students marvel at images of planetary nebulae that spew their contents in diametrically opposed jets of gas and at elegant spiral galaxies (among the most beautiful objects in the universe). We finish with images of the deep field survey of galaxies. Few images are 
more stirring-galaxy upon galaxy upon galaxy piled up one after the other to the edges of the universe, each galaxy possessing millions, billions or even trillions of stars. If a goal of general education is to imbue a sense of place and scale in our students, what could be more effective than a deep understanding of the universe? On the other hand, it is not our intention to make them feel small and insignificant. As an antidote to this natural feeling of being miniscule, the following quote is read from Alan Dressler's 1994 book, Voyage to the Great Attractor (Dressler 1994).

We continue to take the wrong lesson from what we are now learning. An astronaut who had taken a tethered spacewalk while on a Gemini flight was recently asked whether the experience had changed him. He had been struck, he recalled, by how small and insignificant were the Earth and the human adventure, "like an ant crawling across the Sahara Desert." Exactly. The ant, astronomically outnumbered by the grains of sand, overwhelmed by the size of the inhospitable desert, is nevertheless the greater marvel, by far.

It is time to take stock of the discovery that life is the most complex thing we know of in the Universe, and, as such, most worthy of our admiration. Yes, the Universe dwarfs our world in size and immense power. But the Universe of stars, galaxies, and vast gulfs of space is so very, very simple compared to us and our brethren life forms. If we could but learn to look at the Universe with eyes that are blind to power and size, but keen for subtlety and complexity, then our world would outshine a galaxy of stars. Indeed, we should marvel at the Universe for its majesty, but we must truly be in awe of its greatest achievement-life.

Granting the statement is more metaphor than science, the origin and evolution of life is certainly an achievement worth exploring in a general education science class. Moreover, this course enables us to frame that grand story in the larger physical and chemical evolutionary context in a way that pure biology courses cannot. Living organisms are made of particles, arranged in atoms to form different elements, which are arranged in a dizzying array of molecules interacting according to their chemical properties. How then did these chemicals originate and how did they come to be on Earth? The first item that we tackle in this last unit is to define life. Like so much in biology, it is more complex than it seems at first. Trefil and Hazen (2007) handle the questions by listing characteristics that most known living organisms share:

1. All living things maintain a high degree of order and complexity.

2. All living things are part of a larger system of matter and energy.
3. All life depends on chemical reactions that take place in cells.

4. All known life requires liquid water.

5. Organisms grow and develop.

6. Living things regulate their use of energy and respond to their environment.

7. All things share the same genetic code, which is passed from parent to offspring.

8. All living things are descended from a common ancestor.

The first two items are concepts that directly relate to earlier discussions of the laws of thermodynamics. One should be careful to point out that these two items do not imply that life violates the second law of thermodynamics, but that living systems are open systems, can receive energy, and are thus free to experience localized increases in order. A bit more time is spent with item 3. First, we survey the stunning diversity of life on the planet. Next, we discuss the complexity of the shapes, sizes, and the even greater diversity of chemical reactions that take place in living systems (Table 1 Chemistry). Despite the complexity of life and its biochemistry, every element on the periodic table is not equally represented in the makeup of living organisms. Indeed, no element is more central to the understanding of life than carbon. If there is such great complexity of shape and chemistry in living systems, then there had better be a flexible building block; the ultimate erector set if you will. Carbon is special. Its ability to form stable covalent bonds with four other atoms, including another carbon atom, makes very large, complex molecules possible. The story of the origins of life must, therefore, tackle an even more fundamental question. If life depends on the chemistry of carbon, where did carbon come from? In answering that fundamental question, we also discover the origin of four other elements from that oft-cited mnemonic from introductory biology classes, CHiNOPS: carbon, hydrogen, nitrogen, oxygen, phosphorus, and sulfur (hydrogen formed during the first second of The Big Bang), all of which are essential to known life and are found as a fairly large percentage of dry biomass. That is why biologists refer to these as essential macroelements. In this course, we show students some impressively complex organic molecules in each of the following types of compounds: lipids, proteins, carbohydrates, and nucleic acids, and some instructors incorporate a lab using molecular models or molecular modeling software.

There are also a number of microelements that are essential, but in much smaller quantities. These include some heavier elements like iron, iodine and zinc. Where do all of the essential elements come from? In the Reassembling the Universe unit, we examine the processes of star birth and death. Students learn that all main sequence stars 
the size of our sun and larger eventually undergo helium fusion resulting in the formation of carbon. Larger stars exhibit fusion layers leading to elements even more massive than carbon, including phosphorus and nitrogen. Indeed, main sequence stars at least eight times larger than our sun will eventually have a core that leads to iron. No amount of pressure in the core of any star can get iron to fuse, so that is the heaviest element formed in the cores of stars. Students can identify and to a large degree explain the processes that lead to the origin of carbon and nearly every other essential element-fusion in star cores. But there are still some outstanding questions the students must ponder. First, some essential elements are more massive than iron, for example iodine. Where do elements heavier than iron come from? Second, how did carbon and all of the other essential elements get to our Solar System?

The answer to both questions is the same-supernova. In Type I supernovae, a white dwarf (a hot carbon/oxygen remnant of sun-sized star) rapidly draws the gas from its binary partner, leading to a rapid accumulation of mass and pressure and, ultimately, the detonation of the former white dwarf (Trefil and Hazen 2007). In Type II supernovae, a large star's iron ash core catastrophically collapses into a neutron core, which rebounds out and smashes into billions of kilometers of in-falling mass. Either way, the energy is monstrous. The energy produced in a supernova of either type exceeds the energy emitted at that moment by all of the stars in a galaxy. The energy generated in a supernova is sufficient to fuse every element of the periodic table, including iodine and other heavier bioessential elements. After the supernova, gases seeded with every element on the periodic table are hurled at a significant portion of the speed of light. This means that at some time in the past, a supernova blasted materials to where our solar system now resides. Those gases either formed or added to a nebula, a huge cloud of gas and debris, that eventually led to our Solar System. Therefore, by carefully developing the sequence of events that lead to red giant and ultimately to a supernova, students now know where all elements, including carbon, came from and how they got to where the Solar System currently resides. Although we have not even come close to explaining the origins of life, we have at least explained the origins of the elements that comprise life, and furthermore, how they got to Earth's neighborhood.

It is one thing to place carbon and all other essential elements in a nebula. It is quite another to locate them on a planet. How, then, did these elements wind up on Earth? Astronomers normally invoke some version of the Nebular Hypothesis. In this hypothesis, nebular gases deep in the cloud, dominated by hydrogen and helium begin to gravitationally contract. But rather than simply contracting into a ball, the cloud begins to develop rotational movement. This rotation spews out thin disks of matter.
Although thin relative to the central mass and ultimate source of the sun's mass, it is still substantial enough to form localized collections of mass due to gravity. Earth is one of these masses that formed in the disc of our developing sun. Like its three inner solar system companions, Earth formed as a smallish rocky planet possessing all of the bioessential elements.

At this point in the class we have established how carbon and all elements wound up on Earth. However, for life to form, we need to explain the origins of organic chemicals and, moreover, the presence of chemicals in the atmosphere. Trefil and Hazen (2007) suggest that we consider what early Earth was like after it formed around our young sun (Table 1 Earth Science). Although eight distinct planets formed (sans Pluto!), there were many, many planetesimals in the early Solar System. These planetesimals ranged from the size of boulders to several kilometers across. Earth is small compared to the sun, but it was large enough to gravitationally entrain many planetesimals. As they smashed into the Earth over and over, the solid Earth became semiliquid and denser materials sank to the core. Over time, Earth cleared its planetary neighborhood of most debris; indeed, that is part of the accepted definition of a planet (Resolution 6 from the XXVIth General Assembly in Prague: Definition of a Planet in the Solar System 2006). As less energy impacted Earth, the great bombardment ended and it cooled. A crust formed, which trapped gases underneath. Gases eventually built up pressure and erupted in global volcanoes. If today's volcanoes are any guide, they spewed simple gases including carbon dioxide, hydrogen, methane, ammonia and especially water vapor. As Earth cooled, atmospheric water condensed and fell as rain filling oceans with rain water. Lightning surely laced this early roiling, charged atmosphere. By this point, we have only established a plausible mechanism for locating simple carbon compounds in the atmosphere, but not producing complex organics. We must still address a simple question - how did carbon get into organic compounds? We can never go back in time, but we can try and reconstruct what the conditions were like at that time. That is what Stanley Miller and Harold Urey did in their famous experiments in the 1950s that showed how the conditions of early Earth could lead to formation of organic molecules (Miller 1953; Miller and Urey 1959). No magic need be invoked, no tricks of modern chemistry introduced, just a simple system of gases, water and heat. There may be other ways that organic compounds could be introduced to early Earth; for instance, meteorites may contain organic compounds that can survive impact on Earth. However it happened, Earth is and surely was rich in organic molecules - a veritable organic soup.

How this soup yielded life is one of the great unresolved mysteries of science, and students learn about the current 
leading theories (Table 1 Life Sciences). Perhaps it had something to do with the way that phospholipids form hollow balls containing mixtures of organic chemicals, perhaps it has something to do with the way RNA molecules can act as both template or enzyme or perhaps it involves myriad processes that we have yet to uncover. The honest scientific stance is that we do not know the answer. But we remind students to relish the unknown, for it is the unknown that drives scientists to journey with stars and to discover. Once life formed, the grand process of evolutionary biology could play out, with natural selection leading the way. The course concludes with an overview of the fossil record and biochemical evidences that support the theory of biological evolution. As Charles Darwin famously said in the end of his book, "There is grandeur in this view of life, with its several powers, having been originally breathed into a few forms or into one; and that, whilst this planet has gone cycling on according to the fixed law of gravity, from so simple a beginning endless forms most beautiful and most wonderful have been, and are being, evolved" (Darwin 1972). We submit that Charles Darwin would be much impressed and deeply moved to see that his version of origins had been moved back to the beginning of time and matter.

\section{The Evolution of Ideas in Science}

Our understanding of the universe has evolved over time as new data are collected, new observations are made, and new explanations are proposed. The primary textbook for the course, The Sciences, is structured around "great ideas" in science that provide a framework for our understanding of the universe (Trefil and Hazen 2007). In the context of these core ideas, the text integrates knowledge from every science discipline in describing the origin, evolution, and nature of the universe and life within it. A major goal is to enable students to understand the fundamental concepts and principles of nature that are common threads in all the sciences, and to be able to apply those concepts to future applications of science that they may encounter. Table 1 lists some of the great ideas in science considered in the course, and described below are examples of how the course traces the evolution of our scientific knowledge regarding the structure of matter and chemistry. These are highlighted here because an understanding of the basic principles of matter and energy is the foundation for comprehending the universe and its evolution.

Structure of Matter An example of the case study approach used in the course involves the evolution of scientific ideas regarding matter. As far as we know, the earliest concept of an atom dates to Democritus of ancient Greece. His philosophical reasoning led him to conclude that there must be a smallest "un-cuttable" (a-tom) piece of any given substance. At the start of the nineteenth century, no one had any defensible ideas about the structure of matter or how elements combine to make compounds. The theory that answered these questions came from John Dalton. He reasoned that if only certain specific ratios of substances combine, it must be due to fundamental units of matter, the atoms, which combine in that same ratio. Some of his original concepts were modified by subsequent scientists to account for new observations, but his basic idea led to numerous discoveries that provided a better understanding of the nature of matter.

Although most nineteenth century scientists accepted the principle that the chemical elements consist of atoms, they knew virtually nothing about the atoms themselves. In 1815 Prout proposed that all atoms consist of multiples of hydrogen atoms. This theory ran into difficulty later in the century when atomic masses were found not to be integer multiples of the mass of a hydrogen atom. In 1869 Dmitri Mendeleev conceived the Periodic Table of the Elements that enabled chemists to predict the properties of thenunknown elements. Discovery of the electron by J.J. Thomson in 1897 led to the realization that all atoms contain electrons. This was the first important insight into the structure of the atom itself. Thomson subsequently proposed his "plum pudding" model of the atom, but that model could neither predict nor explain observed atomic spectra such as the Balmer spectrum of hydrogen. The groundbreaking alpha particle scattering experiment by Ernest Rutherford in 1911 was the prototype of all present day probing of the atom using particle accelerators. This experiment showed that Thomson's model was incorrect and it led Rutherford to propose his theory of the "nuclear atom".

However, the nuclear model of the atom also hit a snag because well-established electromagnetic theory predicted that atoms conforming to this model should collapse. The apparent disparity between observation and theory ushered in the scientific revolution called the "quantum theory of the atom". In 1913 Neils Bohr proposed the quantum model to explain the hydrogen spectrum, and for the first time, there was a theory that precisely predicted the wavelengths of all the hydrogen spectral lines. Bohr found it necessary to invoke quantum postulates that broke sharply from classical physics. The atomic electrons could only occupy discrete quantized energy levels, which meant they could only move in certain "allowed" orbits. Wolfgang Pauli later used quantum physics to explain the periodicity of the periodic table and the existence of inert elements, and revealed the principle that underlies the building up of the elements in the periodic table. 
Chemical Change Combustion was the first chemical change to be studied intensively. Aristotle, who gave the first non-supernatural explanation, hypothesized that all flammable material contained earth and fire as constituents. In the eighteenth century, the phlogiston hypothesis extended Aristotle's idea to other reactions, and the word "fire" was replaced by the more esoteric term "phlogiston" (Greek word for flame). The escape of phlogiston from matter was postulated as the explanation for the loss of mass observed when most substances are burned. The phlogiston hypothesis ran into serious trouble; however, when it was shown that when a metal is heated until it turns to a powder, the powder weighs more than the metal. Proponents of the phlogiston hypothesis were forced to assume that phlogiston could sometimes have negative weight.

However, scientists evolved new ideas to explain the observed weight changes caused by combustion. Antoine Lavoisier conducted a series of experiments that demolished the phlogiston hypothesis. He showed that heating a metal to form a powder requires a substance from the air. By heating a piece of tin floating on a block of wood, and covered by a glass jar, he showed that one-fifth of the air combined with the tin. When Joseph Priestly discovered oxygen, Lavoisier realized that this was the gas that combined with substances upon heating. Not only did this provide a satisfactory explanation for combustion processes, but it also led to a very important idea that governs all chemical changes: the Law of Conservation of Matter. Subsequent scientists would broaden the principle of Conservation of Matter to develop the Law of Conservation of Energy, and Einstein would put forth the remarkable idea that matter could be converted into energy according the equation $E=m c^{2}$. Extensions of these great ideas regarding matter and energy include the concept that electromagnetic radiation has not only properties of waves (energy), but also properties of particles (matter); DeBroglie put forth the extraordinary idea that matter has wave properties. Hence, the overthrow of the phlogiston hypothesis was an impressive chapter in the history of ideas. In its day, there was no more respected concept in all of chemistry, but its demise was caused by and led to the development of new great ideas with far-reaching implications.

Scientists today have a good grasp of atomic and molecular structure and bonding as well as the processes leading to chemical changes, and have learned to "evolve" chemicals through synthesis to suit a variety of purposes. New frontiers beckon as we now seek to understand supramolecular assembly of molecules into layers, cages, helices, and other geometric possibilities with novel properties. By establishing the rules by which molecules self-assemble both in nature and in the laboratory flask, we can design new molecular architectures, predict the properties of new supramolecular structures, and contribute to the continuing evolution of chemistry and the structure of matter. This new knowledge also provides the foundation of a better understanding of the chemical and physical processes that led to the origin of life.

\section{Resources}

The corresponding laboratory manual for The Sciences uses common lab equipment to give students first-hand experience with the scientific process (Stanionis 1998). The labs most relevant to the topics taught in Science and the Concept of Evolution are related to prediction and measurement in science, scientific models and modeling, the acceleration due to gravity, the Laws of Thermodynamics, electromagnetic wavelengths and spectra, stellar evolution, heredity and probability, and natural selection.

High school teachers may offer a similar curriculum for their classes through the Voyages Through Time curriculum series (SETI Institute 2005). It provides textual and visual materials for teachers and students to explore concepts of evolution from the beginnings of the universe to the development of modern technology through six modules: Cosmic Evolution, Planetary Evolution, Origin of Life, Evolution of Life, Hominid Evolution, and Evolution of Technology. The accompanying "Student Reader" books for each of the six modules include journal and magazine articles from the current literature. Throughout the curriculum, students consider three major questions: (1) What is changing? (2) What are the mechanisms for change? (3) What is the rate of change? The major goals of the curriculum are for students to understand that:

- "Evolution is the result of cumulative changes over time that occur in all realms of the natural world.

- Various processes underlie these changes in both the physical universe and in living systems.

- There are differing time scales and rates of change.

- There are connections and relationships between the physical universe and the life it hosts.

- Science is a process of advancing our understanding of the natural world, not a set of final answers or beliefs (SETI Institute 2005).”

Instructors could modify the Voyages Through Time curriculum for use with non-science majors at the college level or for use with middle school students.

Most of the labs and demonstrations listed on the syllabus are found in the lab manual for The Sciences (Stanionis 1998) or in standard introductory chemistry, physics, and biology lab books. Additional print and media resources are listed below. 
Acknowledgment This paper was prepared for a presentation given at the 233rd National Meeting of the American Chemical Society (ACS) in March 2007 at the symposium "Chemical Evolution I: Chemical Change Across Space and Time". The symposium was coorganized by Lori Zaikowski and Jon M. Friedrich, and was cosponsored by the ACS Divisions of Chemical Education (CHED), Geochemistry, and Nuclear Chemistry and Technology. We thank the following for providing financial support for the symposium, attendees, ACS Symposium Series book "Chemical Evolution Across Space and Time", and DVD production: CHED, Dowling College, Fordham University, The Meteoritical Society, and NSF (Award No. 03-35799).

\section{References}

Trefil J, Hazen RM. The sciences: an integrated approach. New Jersey: Wiley; 2007.

Livio M. The accelerating universe: infinite expansion, the cosmological constant, and the beauty of the cosmos. New York: Wiley; 2000.

Glanz J. Cosmic Motion Revealed. Science 1998;282:2156-2157.

Dressler A. Voyage to the great attractor: exploring intergalactic space. New York: A.A. Knopf; 1994.

Resolution 6 from the XXVIth General Assembly in Prague: definition of a planet in the solar system, 2006. International Astronomical Union. http://www.iau.org/resolution_at_GAXXVI.340.0.html (accessed March 17, 2007).

Miller SL. A production of amino acids under possible primitive Earth conditions. Science 1953;117:528-9.

Miller SL, Urey HC. Organic compound synthesis on the primitive Earth. Science 1959;130:245-51.

Darwin C. The origin of species by means of natural selection: or the preservation of favored races in the struggle for life. New York: AMS Press; 1972.
Stanionis VA. Laboratory manual for the sciences: an integrated approach. New Jersey: Wiley; 1998.

SETI Institute. Voyages through time. Santa Cruz, CA: Learning in Motion; 2005.

Books

Chalmers AF. What is this thing called Science? Saint Lucia, Queensland: University of Queensland Press; 1976.

Edey MA, Johanson DC. Blueprints: solving the mystery of evolution. New York: Penguin Books; 1989.

Krauskopf KB, Bieser A. The physical universe. New York: McGrawHill; 1991.

SETI Institute. Voyages through time. Six high school curriculum modules. Santa Cruz, CA: Learning in Motion; 2005.

White FD (1989) Science and the human spirit. Belmont, CA: Wadsworth.

\section{VHS and DVD}

Creation of the Universe, PBS Home Video \# 135, 1990.

Evolution (Darwin \& Natural Selection), Hawkhill Associates, 125 East Gilman St., Madison, WI 53703.

Evolution: Constant Change and Common Threads, Howard Hughes Medical Institute, 400 Jones Bridge Road, Chevy Chase, MD 20815, 2005. URL http://www.biointeractive.org (accessed April $15,2007)$

Powers of Ten, The Films of Charles and Ray Eames, Volume 1, Pyramid Film \& Video: Santa Monica, CA, 90404, 1989.

Voyages Through Time, Six DVD modules. Learning in Motion, Inc.: Santa Cruz, CA, 2005. URL http://www.voyagesthroughtime.org (accessed March 17, 2007). 